\title{
Moving a TAD closer to unravelling chromosome architecture
}

During interphase, the chromosomes of animal cells are organized into regions with high intra-chromosomal interactions, which are known as topologically associating domains (TADs), and regions of low or no intra-chromosomal interaction, which are known as inter-TADs. Two new studies reveal a correspondence between this level of chromatin organization and cytogenetic features in Drosophila melanogaster cells and shed light on the mechanisms of chromatin folding and their relationship with gene expression.

Polytene chromosomes are typically found in the salivary glands of some insect larvae, although they can also be present in other insect tissues and in non-insect species. Polytene chromosomes occur as a result of several rounds of DNA replication without cell division, with the sister chromatids remaining aligned to each other. Dense chromatin regions, known as bands, alternating with less dense regions, known as interbands, are visible when these large chromosomes are observed by light microscopy.

Eagen et al. generated TAD profiles by performing chromosome conformation capture followed by high-throughput sequencing $(\mathrm{Hi}-\mathrm{C})$ in salivary gland cells of $D$. melanogaster larvae. The genome-wide polytene $\mathrm{Hi}-\mathrm{C}$ heatmaps thus produced were then superimposed onto previously published sequence-annotated maps of the locations of polytene bands in this species. Ulianov et al. performed $\mathrm{Hi}-\mathrm{C}$ in four D. melanogaster cell lines from different lineages and developmental stages and then compared previously published interband profiles with the TAD profiles obtained from the $\mathrm{Hi}-\mathrm{C}$ analysis. Both groups found that TADs correspond to polytene bands, whereas inter-TADs correspond to interbands. The findings of Ulianov et al. show that this relationship is mostly conserved across different tissues and developmental stages. Eagen et al. analysed their data in combination with previously published $\mathrm{Hi}-\mathrm{C}$ maps of D. melanogaster diploid cells and also saw that TAD profiles are mostly conserved between polytene and diploid cells. The two studies show that TADs are mostly (albeit not always) inactive chromatin regions, whereas inter-TADs are areas of active transcription, containing many housekeeping genes.

How are TADs and inter-TADs formed? On the basis of their analysis of active and inactive chromatin marks and computer simulations of the folding of a linear polymer, Ulianov et al. propose that TADs are generated through a self-organization process. Nucleosomes from inactive chromatin would associate with other nucleosomes, with each nucleosome sticking to a single other nucleosome to prevent aggregation of all nucleosomes. Active chromatin marks such as acetylation and the presence of RNA polymerase II - would perturb the interaction between nucleosomes and thereby the formation of TADs. Transient expression of a non-housekeeping gene (for example, in a specific tissue or during a particular period of development) would temporarily disrupt the formation of a TAD.

Eagen et al. analysed the degree of chromatin compaction in bands and interbands of polytene chromosomes. They found two types of euchromatin, one that is 10 -fold more condensed than the other, and heterochromatin, which is condensed up to 30 -fold more than open chromatin. The researchers propose that inactive TADs are regions of heterochromatin; that inter-TADs contain enhancers and promoters and are regions of fully extended euchromatin; and that active TADs contain the coding regions of genes that are being actively transcribed. Given the known distribution of heterochromatin and euchromatin in the nucleus, inactive TADs would be typically localized to the periphery of the nucleus, whereas inter-TADs and active TADs would be located to the middle of the nucleus, according to this model.

The new findings establish a link between direct visual observation of the organization of the nucleus at interphase and the molecular structure of chromatin. They also provide novel insights into the functional links between the mechanisms of chromatin folding and gene expression.

Joana Osório The author declares no competing interests. This article is modified from the original in Nat. Rev. Genet. (http://dx.doi.org/10.1038/nrg.2015.7)

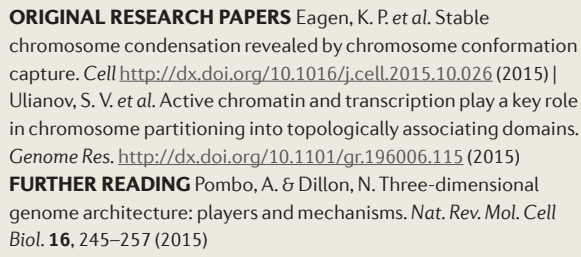

